\title{
Robotic-assisted Laparoscopic Prostatectomy: Initial Experience of 267 Cases
}

\author{
(1) Ekrem İslamoğlu MD, ( Y Yasin Aktaş MD, ( Özgür Arı MD, • Hakan Anıl MD, ( Ali Yıldız MD, ๑ Mutlu Ateş MD, \\ (D) Murat Savaş MD
}

University of Health Sciences, Antalya Training and Research Hospital, Clinic of Urology, Antalya, Turkey

\begin{abstract}
Objective: To present our experience of 267 consecutive patients treated with robotic-assisted laparoscopic prostatectomy (RALP) and assess the perioperative and postoperative outcomes.

Materials and Methods: We retrospectively analyzed the data of 267 men who underwent RALP in our clinic between March 2015 and April 2018. Preoperative clinical data including age, serum prostate-specific antigen (PSA), biopsy Gleason score, and number of positive cores were noted. Perioperative parameters such as operative time and intraoperative complications were recorded. Postoperative parameters including hematocrit change, length of hospital stay, and catheter removal date were noted. Pathological outcomes included pathological Gleason score; positive surgical margin (PSM) status; extracapsular, lymphovascular, perineural, and seminal vesicle invasion; and lymph node positivity. The Clavien-Dindo system was used to classify surgical complications.

Results: The mean age of the patients was $64.2 \pm 6.4$ years and the median PSA was $8.27 \mathrm{ng} / \mathrm{dL}$. The mean operative time was $196.4 \pm 59.4$ min and median hematocrit decrease was 3.9\%. The overall PSM rate was $21.34 \%$ and this rate increased significantly with final pathological stage from $12.97 \%$ for $\mathrm{pT} 2$ to $35.48 \%$ for $\mathrm{pT} 3$ ( $\mathrm{p}<0.05)$. Over a mean follow-up time of 19 months, biochemical recurrence occured in 29 patients $(9.7 \%)$ and a total of 35 patients (22\%) required additional treatment. A total of 29 patients (10.86\%) had complications and 1 patient required surgical intervention in the first 48 hours after surgery. The median postoperative hospital stay was 3 days and median time to urethral catheter removal was 10 days.

Conclusion: Our initial experience with RALP is promising. Oncological outcomes were satisfactory, with patients benefiting from the advantages of the minimally invasive surgical approach.
\end{abstract}

Keywords: Prostate cancer, robotic-assisted laparoscopic prostatectomy, outcomes

\section{Introduction}

Prostate cancer (PCa) is the commonest cancer in males in the United States and the second leading cause of cancer deaths (1). In Turkey, it is the second most common cancer in all age groups and affects $11 \%$ of men (2). Radical prostatectomy remains the gold standard surgical treatment for localized PCa. Robotic-assisted laparoscopic prostatectomy (RALP) was first reported by Binder et al. (3) in 2000 and became widely used all around the world. RALP has many advantages over open and pure laparoscopic radical prostatectomy. Threedimensional magnified vision, enhanced ergonomics, and the use of an endo-wrist instrument with seven degrees of freedom in range of motion are the main advantages. High costs, inability to understand tissue or suture tension due to lack of tactile sensation, and collision of robotic arms with each other or the assistant port are disadvantages of this technique (4). In this study, we present our experience with 267 consecutive RALP procedures and assess the perioperative and postoperative outcomes.

\section{Materials and Methods}

Data pertaining to 267 men who underwent RALP in our clinic between March 2015 and April 2018 were evaluated retrospectively.

All RALP procedures were performed via transperitoneal approach using 6 trocar ports and a conventional 4-arm da Vinci XI robotic system. We began with initial dissection of the seminal vesicles and the prostate in a posterior fashion, 
then returned to the anterior aspect of the prostate and separated the dorsal vein complex. The neurovascular bundle (NVB) was completely released and the prostate was dissected from the bladder neck. Urethrovesical anastomosis was done continuously using two $15 \mathrm{~cm} 3-0$ V-lock sutures, and an 18-French Foley catheter with $10 \mathrm{~mL}$ balloon was inserted. Bilateral pelvic lymphadenectomy (BPLND) was performed in all high-risk and selected intermediate-risk patients according to Briganti et al. (5)'s nomogram. Preoperative clinical data including age, serum prostate-specific antigen (PSA), biopsy Gleason score, and number of positive cores were noted. Perioperative parameters such as operative time, intraoperative complications, and whether BPLND or NVB preservation was done were recorded. Operative time was defined as skin-toskin time in minutes and includes docking and undocking time. Postoperative parameters including change in hematocrit, length of hospital stay, and time to catheter removal were noted. Pathological outcomes included pathological Gleason score; positive surgical margin (PSM) status; extracapsular, lymphovascular, perineural, and seminal vesicle invasion; and lymph node positivity. The Clavien-Dindo system was used to classify operative complications (6).

Written informed consent forms were obtained from each patient and the study was conducted in accordance with the Declaration of Helsinki. Routinely collected patient data in the database was analyzed retrospectively to evaluate clinical and pathological outcomes. Ethics committee approval was not sought because the study also included retrospective data.

\section{Statistical Analysis}

Basic and descriptive statistical analyses were used in this study and all data were expressed as mean or median (minimum, maximum) for numerical variables and as frequencies and percentages for categorical variables. Statistical analyses were done using IBM SPSS Statistics for Windows, Version 22.0 (IBM Corp., Armonk, NY).

\section{Results}

The mean age of the patients was $64.2 \pm 6.4$ years and the median PSA was $8.27 \mathrm{ng} / \mathrm{dL}$ (range 0.3-53.4). Preoperative clinical characteristics and perioperative outcomes are shown in Table 1. Skin-to-skin operative time ranged from 174 to $410 \mathrm{~min}$ and the median hematocrit decrease was $3.9 \%$ (range $0.5-14.5 \%$ ). The overall PSM rate was $21.34 \%$ and this rate increased significantly with final pathological stage from $12.97 \%$ for $\mathrm{pT} 2$ to $35.48 \%$ for $\mathrm{pT} 3(\mathrm{p}<0.05)$. Pathological results and clinical outcomes are shown in Table 2. During a mean follow-up of 19 months, 26 patients (9.7\%) received adjuvant radiotherapy to the prostatic fossa due to the biochemical recurrence and 11 patients (4.1\%) with lymph node positivity received early adjuvant hormone therapy. Complications are shown in Table 3. A total of 29 patients (10.86\%) had complications (each with a single event) and 1 of them $(1 / 267)$ required surgical intervention in the first 48 hours after surgery due to ileum perforation. Six patients with urethra-vesical anastomosis stenosis and four patients with urethral stricture were treated with endoscopic intervention. The median postoperative hospital stay was 3 days (range 2-7 days) and median time to urethral catheter removal was 10 days (range 10-14).

\begin{tabular}{|c|c|c|}
\hline \multicolumn{2}{|l|}{ Variable } & Result \\
\hline \multicolumn{2}{|l|}{ Age, years (mean $\pm S D$ ) } & $64.2 \pm 6.4$ \\
\hline \multicolumn{2}{|l|}{ PSA, ng/dL median (min-max) } & $8.27(0.3-53.4)$ \\
\hline \multicolumn{2}{|c|}{ Number of cores positive, median (min-max) } & $3.72(1-12)$ \\
\hline \multirow{3}{*}{ Biopsy Gleason score, n (\%) } & $4-6$ & $172(64.4)$ \\
\hline & 7 & $82(30.7)$ \\
\hline & $8-10$ & $13(4.9)$ \\
\hline \multirow{3}{*}{ Risk group, n (\%) } & Low & $137(51.3)$ \\
\hline & Intermediate & $94(35.2)$ \\
\hline & High & $36(13.5)$ \\
\hline Operating time, minutes (mean \pm SD) & - & $196.4 \pm 59.4$ \\
\hline $\begin{array}{l}\text { Hematocrit decrease, \% median } \\
\text { (min-max) }\end{array}$ & - & $3.9(0.5-14.5)$ \\
\hline BPLND, n (\%) & - & $83(31.1)$ \\
\hline NVB preservation, n (\%) & - & $53(19.8)$ \\
\hline
\end{tabular}
lymphadenectomy, NVB: Neurovascular bundle, Min: Minimum, Max: Maximum

\begin{tabular}{|c|c|c|}
\hline \multicolumn{2}{|l|}{ Variable } & Result \\
\hline \multirow{2}{*}{ Surgical margin status, n (\%) } & Positive & $210(78.7)$ \\
\hline & Negative & $57(21.3)$ \\
\hline \multirow{2}{*}{ Extracapsular invasion, n (\%) } & Yes & $82(30.7)$ \\
\hline & No & $185(69.3)$ \\
\hline \multirow{2}{*}{ Lymphovascular invasion, n (\%) } & Yes & $50(18.7)$ \\
\hline & No & $217(81.3)$ \\
\hline \multirow{2}{*}{ Perineural invasion, $\mathrm{n}(\%)$} & Yes & $203(76.0)$ \\
\hline & No & $64(24.0)$ \\
\hline \multirow{2}{*}{ Seminal vesicle invasion, $\mathrm{n}(\%)$} & Yes & $38(14.2)$ \\
\hline & No & $229(85.8)$ \\
\hline \multirow{3}{*}{ Pathological Gleason score, n (\%) } & $4-6$ & $106(39.7)$ \\
\hline & 7 & $141(52.8)$ \\
\hline & $8-10$ & $20(7.5)$ \\
\hline \multirow{2}{*}{ Lymph node positivity, n (\%) } & Yes & $11(13.2)$ \\
\hline & No & $72(86.8)$ \\
\hline \multirow{2}{*}{ Pathological stage, n (\%) } & pT2 & $201(75.3)$ \\
\hline & pT3 & $66(24.7)$ \\
\hline \multirow{2}{*}{ Biochemical recurrence, $\mathrm{n}(\%)$} & Yes & $26(9.7)$ \\
\hline & No & $241(90.3)$ \\
\hline \multicolumn{2}{|l|}{ Duration of follow-up, months } & $19(3-37)$ \\
\hline \multicolumn{2}{|l|}{ Additional treatment, n (\%) } & $37(13.8)$ \\
\hline
\end{tabular}




\begin{tabular}{|l|l|l|l|}
\hline $\begin{array}{l}\text { Table 3. Classification of complications using the Clavien-Dindo } \\
\text { system }\end{array}$ & $\mathbf{n}$ & $\%$ & $\begin{array}{l}\text { Clavien-Dindo } \\
\text { grade }\end{array}$ \\
\hline Complication & & & \\
\hline Intraoperative & 2 & 0.74 & Grade 4 \\
Ureteral injury & 1 & 0.37 & Grade 4 \\
Ileum perforation & 2 & 0.74 & Grade 4 \\
Vascular injury & 1 & 0.37 & Grade 2 \\
Tachycardia & & & \\
\hline Postoperative & & & \\
Urethra-vesical anastomosis stenosis & 6 & 2.24 & Grade 3 \\
Lymphosel (required drainage) & 2 & 0.74 & Grade 3 \\
Lymphosel (not required drainage) & 4 & 1.49 & Grade 2 \\
Urine leakage & 2 & 0.74 & Grade 2 \\
Urethral stricture & 4 & 1.49 & Grade 3 \\
\hline Medical & & & \\
Wound infection & & & \\
Transfusion & 2 & 0.74 & Grade 2 \\
\hline Total & 3 & 1.12 & Grade 2 \\
\hline
\end{tabular}

\section{Discussion}

Radical prostatectomy has been a challenging surgery since its introduction in 1905 by Hugh Hampton Young. Due to the deep location of the prostate within the pelvis and its extensive vascularization, radical retropubic prostatectomy has continued to have significant surgical morbidity over the years. The search for less invasive techniques with less blood loss and postoperative pain, shorter hospitalization, and improved quality of life without sacrificing oncological results has led surgeons to learn and perform laparoscopic radical prostatectomy (LRP). Robotic surgery was introduced to overcome the limitations of LRP such as the non-ergonomic instruments, difficulty in urethra-vesical anastomosis and steep learning curve with its three-dimensional magnified vision and endo-wrist instruments.

We reported our first 267 RALP procedures, including the learning curve. It has been suggested that at least 50 cases are needed to gain proficiency in $\operatorname{RALP}(7,8)$. Although this study included the initial experience in robotic surgery our operative time was compatible with the literature $(9,10)$. Perioperative mean estimated blood loss was under the average and only two patients required blood transfusion after the operation. None of the patients were converted to open surgery and two major complications that occurred during surgery were treated with robotic surgery intraoperatively. These promising perioperative results may be attributed to the advanced laparoscopic skills of the surgeons before their experience with the robotic system.

The presence of PSM after radical prostatectomy is an independent risk factor for local recurrence and disease progression (11). This factor can be influenced by surgeon experience and the main goal of any urologist should be to reduce the PSM rate. In the current study, the PSM rate was $21.34 \%$ and this result was consistent with the literature. In the most extensive literature review, Novara et al. (12) reported a $15 \%$ mean rate of PSM in RALP series published between 2008 and 2011 (each including $>100$ cases), with a range of 6.5$32 \%$ and concluded that PSM rate is higher in men with more advanced pathologic stage. Our PSM rates were $12.97 \%$ for pT2 tumors and $35.48 \%$ for pT3 tumors. Lymph node positivity was $13.2 \%$ for patients who underwent BPLND (11/83), which was close to Briganti et al. (5)'s results of $12 \%$ (13). Complications are a troubling aspect of surgical interventions and should be handled carefully. We classified complications according to the Clavien-Dindo system in this study and our $10.8 \%$ complication rate was within the average range when compared with newer series reporting rates from $5.08 \%$ to $19.6 \%(14,15)$. We hope to decrease our complication rates as we increase our experience in RALP.

\section{Study Limitations}

This study has some limitations. Although it was based on a prospective database the study was retrospective. In addition, the follow-up period was relatively short and oncological outcomes such as biochemical recurrence require further observation. We did not compare our RALP results with outcomes of open or pure LRP in our clinic, which may better demonstrate the advantages of robotic surgery. Lastly, the cohort was small, with 267 cases, and a study with a larger sample size may change the results of some parameters.

\section{Conclusion}

Our initial experience with RALP is promising. We were able to transfer our LRP technique to robotic surgery with minimal difficulty. Oncological outcomes were adequate with the patient benefiting the advantages of the minimally invasive surgical approach.

\section{Acknowledgments}

The authors thank Başak Oğuz MD, and appreciate her support in the statistical analysis of this study.

\section{Ethics}

Ethics Committee Approval: Retrospective study.

Informed Consent: Retrospective study.

Peer-review: Externally peer-reviewed.

\section{Authorship Contributions}

Concept: E.I.., M.A., M.S., Design: E.I.,, Data Collection or Processing: Y.A., H.A., Ö.A., Analysis or Interpretation: Y.A., H.A., Ö.A., Literature Search: E.I., Y.A., H.A., Ö.A., A.Y., Writing: E.I.

Conflict of Interest: No conflict of interest was declared by the authors.

Financial Disclosure: The authors declared that this study received no financial support.

\section{References}

1. Siegel RL, Miller KD, Jemal A. Cancer statistics, 2018. CA Cancer J Clin 2018;68:7-30.

2. Gültekin M, Boztaş G. Türkiye kanser istatistikleri. Saglık Bakanlığı, Türkiye Halk Saglıgı Kurumu, 2014:43.

3. Binder J, Kramer W. Robotically-assisted laparoscopic radical prostatectomy. BJU Int, 2001;87:408-410.

4. Kural AR, Atug F. The applications of robotic surgery in urology. Turk J Urol 2010;36:248-257. 
5. Briganti A, Larcher A, Abdollah F, et al. Updated nomogram predicting lymph node invasion in patients with prostate cancer undergoing extended pelvic lymph node dissection: the essential importance of percentage of positive cores. Eur Urol 2012;61:480-487.

6. Clavien PA, Barkun J, de Oliveira ML, et al. The Clavien-Dindo classification of surgical complications: five-year experience. Ann Surg 2009;250:187-196.

7. Ou YC, Yang CR, Wang J, et al. The learning curve for reducing complications of robotic-assisted laparoscopic radical prostatectomy by a single surgeon. BJU Int 2011;108:420-425.

8. Sooriakumaran $\mathrm{P}$, John M, Wiklund $\mathrm{P}$, et al. Learning curve for robotic assisted laparoscopic prostatectomy: a multi-institutional study of 3794 patients. Minerva Urol Nefrol 2011;63:191-198.

9. Tasci Al, Tufek I, Gumus E, et al. Oncologic results, functional outcomes, and complication rates of robotic-assisted radical prostatectomy: multicenter experience in Turkey including 1,499 patients. World J Urol 2015;33:1095-1102.

10. Galfano A, Di Trapani D, Sozzi F, et al. Beyond the learning curve of the Retzius-sparing approach for robot-assisted laparoscopic radical prostatectomy: oncologic and functional results of the first 200 patients with $\geq 1$ year of follow-up. Eur Urol 2013;64:974-980.

11. Shahabi A, Satkunasivam R, Gill IS, Lieskovsky G, et al. Predictors of time to biochemical recurrence in a radical prostatectomy cohort within the PSA-era. Can Urol Assoc J 2016;10:17-22.

12. Novara G, Ficarra V, Mocellin S, et al. Systematic review and metaanalysis of studies reporting oncologic outcome after robot-assisted radical prostatectomy. Eur Urol 2012;62:382-404.

13. Gandaglia G, Fossati N, Zaffuto E, et al. Development and internal validation of a novel model to identify the candidates for extended pelvic lymph node dissection in prostate cancer. Eur Urol 2017;72:632-640.

14. Lasser MS, Renzulli J 2nd, Turini GA 3rd, et al. An unbiased prospective report of perioperative complications of robot-assisted laparoscopic radical prostatectomy. Urology 2010;75:1083-1089.

15. Menon M, Dalela D, Jamil M, et al. Functional recovery, oncologic outcomes and postoperative complications after robot-assisted radical prostatectomy: an evidence-based analysis comparing the Retzius sparing and standard approaches. J Urol 2018;199:1210-1217. 